\title{
SOME APPLICATIONS OF ZORN'S LEMMA IN ALGEBRA
}

\author{
D. D. ANDERSON, DAVID E. DOBBS AND MUHAMMAD ZAFRULLAH
}

\begin{abstract}
We indicate some new applications of Zorn's Lemma to a number of algebraic areas. Specifically, we show that a property $P$ holds for all the subobjects of a given object if and only if $P$ supports both the chain condition from Zorn's Lemma and some finitistic conditions on subobjects that have the flavor of mathematical induction. The specific algebraic contexts considered are modules, sets, groups, algebras, and integral domains. Particular emphasis is given to applications involving star operations on integral domains.
\end{abstract}

The use of Zorn's Lemma has been a part of the mainstream in virtually every area of algebra for more than 75 years. The aim of this note is to indicate some new applications of Zorn's Lemma to a number of algebraic areas by using a slightly different perspective. In Theorem 1 and its aftermath, we show that a property $P$ holds for all the subobjects of a given object if and only if $P$ supports both the chain condition from Zorn's Lemma and some finitistic conditions on subobjects that have the flavor of mathematical induction. Rather than stating our results categorically, we content ourselves with giving a number of reformulations of Theorem 1 for various algebraic settings, thus pointing out some of the venues where our approach may come in handy. In detail, we prove Theorem 1 for modules, give its analogs for sets, groups, algebras, and commutative integral domains and indicate some applications, especially in the context of star operations on integral domains. By a chain, we mean a nonempty collection of subsets that is totally ordered under inclusion. Throughout, all rings are associative with identity and all modules are left unital.

Theorem 1. Let $R$ be a ring and let $E$ be a (left) $R$-module. Let $P$ be a property of modules. Then each nonzero submodule of $E$ satisfies property $P$ if and only if the following three conditions hold.

Corresponding author: David E. Dobbs.

Received February 20, 2008.

2000 Mathematics Subject Classification. Primary 13A15, 13L05; Secondary 13C10, 13C11, 13B24, $13 \mathrm{G} 05$.

Key words and phrases. Zorn's Lemma, submodule, projective, injective, semisimple, flat, finitely generated, subalgebra, going-down, going-up, subgroup, integral domain, star operation, *-Prüfer domain, *-Krull domain, suboboject.

The second-named author was supported in part by a University of Tennessee Professional Leave Award. 
(1) $P$ is true for every nonzero cyclic submodule of $E$.

(2) If $A$ is a nonzero submodule of $E$ such that $A$ satisfies property $P$ and $x \in E$, then $A+R x$ again satisfies property $P$.

(3) If $\left\{H_{\alpha}\right\}$ is a chain of nonzero submodules of $E$ such that each $H_{\alpha}$ satisfies property $P$, then $\cup H_{\alpha}$ satisfies property $P$.

Proof. Suppose that (1), (2) and (3) hold. If the "if" assertion fails, $E$ has a nonzero submodule $A$ such that $A$ does not have property $P$. Let $S$ be the set of all nonzero submodules $I$ of $A$ such that $I$ has property $P$. Then $S$ is nonempty because, by (1), every nonzero cyclic submodule has property $P$. Moreover, $S$ can be partially ordered by inclusion. By (3), $S$ meets the requirements of Zorn's Lemma, and so must contain a maximal element $J$. Since $A$ does not have property $P, J \subsetneq A$. Pick $x \in A \backslash J$. Note that $J+R x \subseteq A$ has property $P$ (by (2)), contradicting the maximality of $J$, thus completing the proof of the "if" assertion. The converse is evident.

Remark 2. In the statement of Theorem 1, we can replace (2) by (2'): If $A$ and $B$ are two nonzero submodules of $E$ such that $A$ and $B$ satisfy property $P$, then $A+B$ satisfies property $P$. We will emphasize the case where $\mathrm{P}$ holds for all nonzero submodules and leave the case where it holds for all nonzero proper submodules to Theorem 3 and the comment that precedes it.

We next indicate that Theorem 1 has an analog for proper modules. Indeed, each of the analogs of Theorem 1 that are developed below in various algebraic contexts has an analog for proper subobjects in an appropriate category. Theorem 3 will provide the statement for proper subobjects in the module-theoretic case. We trust that the interested reader will verify that the proof of Theorem 1 carries over, mutatis mutandis, to yield the proof of Theorem 3 and of the corresponding "proper" analogs of later results.

Theorem 3. Let $R$ be a ring and let $E$ be a (left) $R$-module. Let $P$ be a property of modules. Then each nonzero proper submodule of $E$ satisfies property $P$ if and only if the following three conditions hold.

(1) $P$ is true for every nonzero proper cyclic submodule of $E$.

(2) If $A$ is a nonzero proper submodule of $E$ such that $A$ satisfies property $P$ and $x \in E$ is such that $A+R x$ is a proper submodule of $E$, then $A+R x$ again satisfies property $P$.

(3) If $\left\{H_{\alpha}\right\}$ is a chain of nonzero proper submodules of $E$ such that each $H_{\alpha}$ satisfies property $P$ and $\cup H_{\alpha}$ is a proper submodule of $E$, then $\cup H_{\alpha}$ satisfies $P$.

Some rather obvious candidates for the property $P$ in Theorem 1 are projective, injective, flat, semisimple, torsion free, and divisible. There are many more, such as "is finitely generated". In the following, we include remarks about some of these choices of $P$. 
We note that if $\mathrm{P}$ is "projective", we get, using Theorem 1, a new characterization of " $E$ is a left $R$-module each of whose submodules is projective". (The fact that we restrict to nonzero submodules in Theorem 1 does not interfere because the zero module is free, hence projective, as well as also being injective and flat.) In a similar fashion, we can characterize left $R$-modules all of whose submodules are injective.

Corollary 4. Let $R$ be a ring. Then $R$ is a left hereditary ring if and only if the following three conditions hold.

(1) Every nonzero principal left ideal of $R$ is projective.

(2) The sum of a projective left ideal with a principal left ideal is a projective left ideal.

(3) The union of any chain of projective left ideals of $R$ is a projective left ideal of $R$.

Moreover, if $R$ is commutative such that (2) and (3) hold, then $R$ is hereditary if and only if $R$ is locally a domain and $T(R)$, the total quotient ring of $R$, is von Neumann regular.

Proof. In view of the above comments, the only part that needs proof is the commutative ring case. But that follows from the first assertion because, according to Glaz [13, Theorem 4.2.10], $R$ being locally a domain and $T(R)$ being von Neumann regular is equivalent to every principal ideal of $R$ being projective.

Recall that a (left) $R$-module $M$ is semisimple if $M$ is a direct sum of simple submodules (see, e.g., [5, page 11]). A semisimple module $M$ is characterized by the property that every submodule of $M$ is a direct summand of $M$ [5, Proposition 4.1, page 11]. Of course this characterization does not change if we replace "every submodule" by "every nonzero submodule". Also, as noted in the proof of [5, Proposition 4.1, page 11] (cf. also [4, Theorem 2.3.2]), every submodule of a semisimple module is a semisimple module. Setting $P=$ "is a direct summand of $M$ " in Theorem 1 gets us the following new characterization of semisimple modules.

Proposition 5. An R-module $M$ is a semisimple $R$-module if and only if the following three conditions hold.

(1) Every nonzero cyclic submodule of $M$ is a direct summand of $M$.

(2) If $A$ is a nonzero submodule that is a direct summand of $M$, then for every nonzero cyclic submodule $B, A+B$ is a direct summand of $M$.

(3) If $\left\{H_{\alpha}\right\}$ is a chain of nonzero submodules of $E$ such that each $H_{\alpha}$ is a direct summand of $M$, then $\cup H_{\alpha}$ is a direct summand of $M$.

Proof. That $M$ semisimple implies (1), (2) and (3) is clear from the above comments. For the converse, take $P=$ "is a direct summand of $M$ " in Theorem 1 . Combining this with the fact that the zero submodule is trivially a direct summand of $M$, we have the conclusion that $M$ is semisimple. 
As a semisimple (left Artinian) ring is defined as a ring that is a semisimple module over itself, the above corollary can be restated using left ideals in place of submodules to characterize semisimple rings. Now, as mentioning all the three conditions stated in Theorem 1 can become a mouthful, we have decided to use " $M$ has $(P, c s u)$ " to indicate that the conditions (1), (2) and (3) of Theorem 1, are satisfied for a suitable property $P$ of modules. ( $c$ comes from cyclic in (1), s from sum in (2) and $u$ from union in (3).)

Because a submodule of a semisimple module is again semisimple, we have the following corollary to Theorem 1. A similar assertion holds for any module-theoretic property $P$ which is inherited by all submodules.

Corollary 6. A left $R$-module $M$ is a semisimple $R$-module if and only if $M$ has (P, csu) with $P=$ semisimple.

While a lone direct summand of a left $R$-module may not be an injective submodule, an injective submodule is necessarily a direct summand [5, Proposition 3.4, page 10]. In view of this fact, we state the following result.

Corollary 7. An R-module $M$ is semisimple and injective if and only if $M$ has $(P$, csu) with $P=$ injective.

Proof. That $M$ has $(P, c s u)$ with $P=$ injective implies that $M$ is semisimple follows from the fact that any injective submodule of $M$ is a direct summand of $M$. The rest of the "if" assertion follows from Theorem 1. Conversely, $M$ being injective, every direct summand of $M$ is injective.

A ring $R$ that is an injective (left) module over itself is called self-injective. Any semisimple ring is self-injective [5, Theorem 4.2, page 11]. The reader may use Theorem 1 to develop the $(P$, csu $)$ characterizations of semisimple rings.

Corollary 8. Let $R$ be a commutative ring. Every ideal of $R$ is finitely generated if and only if every union of a chain of finitely generated ideals of $R$ is again a finitely generated ideal of $R$.

The case of Theorem 1 in which $P=$ flat is interesting in that in characterizing flat modules, we do not explicitly need the third condition.

Corollary 9. Let $R$ be a ring and let $E$ be a left $R$-module. Then each nonzero submodule of $E$ is flat if and only if the following two conditions hold.

(1) Every cyclic nonzero submodule of $E$ is flat.

(2) If $A$ and $B$ are nonzero submodules of $E$ with $A$ flat and $B$ cyclic, then the sum $A+B$ is also flat.

Proof. Apply Theorem 1, with $P=$ flat, noting that condition (3) is automatic, since any direct limit of flat modules is flat. 
The above corollary is not new; we found it as part (2) of [19, Proposition 6.11].

The following result, being in the same vein as [5, Theorem 4.2, page 11], talks about all the left (right) ideals of a ring being flat. We include it here as this result does not seem to be as well known as [5, Theorem 4.2, page 11].

Theorem 10.([19, Proposition 6.12]) For a ring $R$, the following conditions are equivalent:

(1) All submodules of flat right $R$-modules are flat;

(2) All submodules of flat left $R$-modules are flat;

(3) All right ideals of $R$ are flat;

(4) All left ideals of $R$ are flat;

(5) All finitely generated right ideals of $R$ are flat;

(6) All finitely generated left ideals of $R$ are flat;

(7) For any finitely generated right ideal $I$ of $R$ and for any finitely generated left ideal $J$ of $R$, the natural homomorphism $I \otimes_{R} J \rightarrow I J$ is an isomorphism;

(8) Every nonzero principal left ideal of $R$ is flat, as is the sum $A+B$ of every pair $A, B$ of nonzero flat left ideals of $R$ where $B$ is cyclic;

(9) Every nonzero principal right ideal of $R$ is flat and the sum of every pair $A, x R$ of nonzero flat right ideals of $R$ is flat.

Proof. The equivalence of (1)-(7) is established in [19, Proposition 6.12] and the equivalence of (8) and (4) (resp., (9) and (3)) can be shown using Corollary 9.

Here is an application of Theorem 1 to commutative integral domains.

Corollary 11. Let $R$ be an integral domain. Then the following three conditions are equivalent:

(a) $R$ is a Dedekind domain (resp., a PID);

(b) The sum of any invertible ideal of $R$ with any nonzero principal ideal of $R$ is invertible (resp., the sum of any two principal ideals of $R$ is principal) and the union of any chain of invertible integral ideals (resp., of nonzero principal integral ideals) of $R$ is invertible (resp., principal);

(c) $R$ is a Prüfer (resp., Bézout) domain such that the union of every chain of invertible integral ideals (resp., of principal integral ideals) of $R$ is invertible (resp., principal).

Proof. We shall deal with the Dedekind, or outside the parentheses part, of the statements and note that the PID, or inside the parentheses part, can be proved similarly.

(a) $\Rightarrow$ (b) is clear, for $R$ is a Dedekind domain if and only if every nonzero ideal of $R$ is invertible. For $(\mathrm{b}) \Rightarrow(\mathrm{c})$, note that $R$ is a Prüfer domain if and only if every nonzero 2 -generated ideal of $R$ is invertible. 
$(c) \Rightarrow(a)$ : Let the property $P$ be "is invertible". Note that every nonzero principal ideal of an integral domain is invertible. It is well known that every invertible ideal is finitely generated, and that every nonzero finitely generated ideal of a Prüfer domain is invertible. So, in a Prüfer domain, the sum of any two invertible ideals is invertible. This takes care of the requirements (1) and (2) of Theorem 1, while requirement (3) is provided by (c). So, by Theorem 1, (c) implies that every nonzero ideal of $R$ is invertible, i.e., that $R$ is a Dedekind domain.

All $R$-algebras $S$ considered below will be commutative and faithful as $R$-modules; i.e., $R \subseteq S$. Thus, an $R$-algebra $S$ is an $R$-module with extra structure, in that $S$ contains $R$ as a subring/subalgebra. This calls for some adjustments as we reformulate Theorem 1 in order to have applications to $R$-algebras. Let us state right away that we shall deal only with unital algebras and subalgebras. By a singly generated $R$-subalgebra of $S$, we mean an $R$-algebra of the form $\langle R, x\rangle$, the intersection of all $R$-subalgebras containing $R$ and the element $x$. In particular, $R$ can be regarded as a singly generated $R$-subalgebra of $R$. Also, let $P$ be a property that makes sense for $R$-algebras. We then have the following algebra-theoretic analog of Theorem 1.

Proposition 12. Let $S$ be an $R$-algebra. Then every $R$-subalgebra of $S$ has property $P$ if and only if the following three conditions hold.

(a) Every singly generated $R$-subalgebra of $S$ has property $P$.

(b) If $T$ and $U$ are $R$-subalgebras of $S$ with property $P$ such that $U$ is singly generated, then $\langle T, U\rangle$, the subalgebra generated by $T$ and $U$, also has property $P$.

(c) If $\left\{H_{\alpha}\right\}$ is a chain of $R$-subalgebras which each have property $P$, then $\cup H_{\alpha}$ also has property $P$.

Proof. The proof of Theorem 1 carries over, mutatis mutandis. In detail, replace "submodule" with "subalgebra" and $J+R x$ with $\langle R, x\rangle$.

In the spirit of Corollary 9, we have numerous applications of Proposition 12. For instance, by taking $P=$ flat, we can characterize $R$-algebras $S$ such that each $R$-subalgebra of $S$ is flat over $R$. For examples of $R$-algebras $S$ satisfying this condition, see the extensions characterized in [11, Theorem 2.4] or the "flat pairs" studied in [1]. The next result gives an additional application of Proposition 12. As usual, GD denotes the going-down property for ring extensions. It is known that GD is preserved by arbitrary direct limits [10, Theorem 2.1].

Corollary 13. Let $S$ be an $R$-algebra. Then $R \subseteq T$ satisfies $G D$ for each $R$ subalgebra $T$ of $S$ (i.e., for each ring $T$ such that $R \subseteq T \subseteq S$ ) if and only if the following two conditions hold.

(1) $R \subseteq T$ satisfies $G D$ for each singly generated $R$-subalgebra $T$ of $S$.

(2) If $T$ and $U$ are $R$-subalgebras of $S$ such that the extensions $R \subseteq T$ and $R \subseteq U$ each satisfy $G D$ and $U$ is singly generated, then $R \subseteq<T, U>$ satisfies $G D$. 
For an example of $R$-algebras $S$ satisfying the conditions in Corollary 13, see the "going-down pairs" studied in [6]. By the way, the analogs of Corollary 13 hold for relatives of the property GD such as GU (going-up), LO (lying-over) and INC (incomparability) since each of these properties is preserved by arbitrary direct limit (see [9, Theorem 2.1] for GU and LO and [10, Proposition 2.3] for INC). For examples of $R$ algebras $S$ illustrating these analogs, see [8] for GU-pairs (and, what ends up being the same thing, LO-pairs) and [7] and [3] for INC-pairs.

For some additional applications of Proposition 12, consider an integral domain $R$ with quotient field $K$. By taking the $R$-algebra $S$ to be $K$, one could apply Proposition 12 for the following three choices of the property $P$ : "is integrally closed", "is flat as an $R$-module", "is a ring of fractions of $R$ ". However, the resulting facts would not be new, for it is known that if $R$ is an integral domain with quotient field $K$, then $R$ is a Prüfer domain if and only if $R[u]$ is integrally closed (resp., a flat $R$-module) for each $u \in K$; and $R$ is a QR-domain if and only if $R[u]$ is a ring of fractions of $R$ for each $u \in K$.

We can also formulate a group-theoretic result that is analogous to Theorem 1

Proposition 14. Let $P$ be a property of groups. Let $G$ be a group. Then every nontrivial subgroup $S$ of $G$ has property $P$ if and only if the following three conditions hold.

(a) Every non-trivial cyclic subgroup of $G$ has property $P$.

(b) If $T$ is a subgroup of $G$ with property $P$ and if $x \in G \backslash\{e\}$, then $\langle T, x\rangle$, the subgroup of $G$ generated by $T$ and $x$, also has property $P$.

(c) If $\left\{H_{\alpha}\right\}$ is a chain of subgroups of $G$ with property $P$, then $\cup H_{\alpha}$ also has property $P$.

The following rather elementary application of Proposition 14 comes from taking $P$ = finite. Thus, a group $G$ is finite if and only if (a) every nontrivial cyclic subgroup of $G$ is finite, (b) if $F$ is a finite non-trivial subgroup of $G$ and if $x \in G \backslash\{e\}$, then $\langle F, x>$ is finite, and (c) if $\left\{H_{\alpha}\right\}$ is a chain of non-trivial finite subgroups of $G$, then $\cup H_{\alpha}$ is a finite subgroup of $G$. Of course, the corresponding statement for an abelian group $G$ follows from Theorem 1 by taking $R=\mathbb{Z}$.

We next formulate an analog of Theorem 1 for sets.

Proposition 15. Let $S$ be a nonempty set and let $P$ be a property of sets. Then every nonempty subset of $S$ has property $P$ if and only if the following three conditions hold.

(a) Every nonempty singleton subset of $S$ has property $P$.

(b) If a nonempty subset $A$ of $S$ has property $P$, then $A \cup B$ has property $P$ for every nonempty singleton subset $B$ of $S$.

(c) If $\left\{H_{\alpha}\right\}$ is a chain of subsets of $S$ and each $H_{\alpha}$ has property $P$, then so does $\cup H_{\alpha}$. 
The proof of Proposition 15 is a straightforward adaptation of the proof of Theorem 1. By taking $P=$ finite, we see that (a) and (b) hold automatically, and so the following assertion is a quick corollary of Proposition 15. A set $S$ is finite if and only $S$ satisfies the ascending chain condition on subsets.

For more results in a somewhat specialized area of commutative ring theory we need the following background information. Let $D$ be an integral domain with quotient field $K$. Further, let $F(D)$ denote the set of nonzero fractional ideals of $D, f(D)$ the set of finitely generated members of $F(D)$, and $I(D)$ the set of nonzero integral ideals of $D$. A star operation on $D$ is a function $*$ on $F(D)$ denoted by $A \mapsto A^{*}$ such that for all $a \in K \backslash\{0\}$ and $A, B, C \in F(D)$, we have $\left(*_{1}\right)(a)^{*}=(a)$ and $(a A)^{*}=a A^{*},\left(*_{2}\right) A \subseteq A^{*}$, $B \subseteq C$ implies $B^{*} \subseteq C^{*}$ and $\left(*_{3}\right)\left(A^{*}\right)^{*}=A^{*}$. For an introduction to star operations, see [12, Sections 32 and 34]. For a given star operation $*$, it is easy to establish that $(A B)^{*}=\left(A^{*} B\right)^{*}=\left(A^{*} B^{*}\right)^{*}(*$-multiplication $)$ and $(A+B)^{*}=\left(A^{*}+B\right)^{*}=\left(A^{*}+B^{*}\right)^{*}$ (*-sum). Call $A \in F(D)$ a $*$-ideal if $A=A^{*}$. Given a star operation *, we can construct $*_{f}$ defined by $A^{*_{f}}=\cup\left\{F^{*} \mid F\right.$ is a finitely generated nonzero subideal of $\left.A\right\}$. A star operation $*$ is said to be of finite type/character if $*=*_{f}$. It is easy to show that $*_{f}$ is of finite type. It is also easy to show that if $*$ is of finite type, $A \in F(D)$ is a $*$-ideal if and only if, for each nonzero finitely generated $F \subseteq A$, we have $F^{*} \subseteq A$. The operation defined on $F(D)$ by $A \mapsto A_{v}=\left(A^{-1}\right)^{-1}$ is a special star operation called the $v$-operation, and the corresponding finite type star operation $v_{f}=t$ is called the $t$-operation. In [14], Heinzer studied integral domains in which every nonzero ideal is a $v$-ideal. This and some recent work [16] led Mimouni [18] to consider the conditions under which every nonzero ideal is a $*$-ideal for a specific, well defined/well known, star operation $*$. Mimouni [18] studied the $t$-operation and the $w$-operation. Here, the $w$-operation is defined by $A \mapsto A_{w}=\left\{x \in K \mid x J \subseteq A\right.$ for some finitely generated $J \in F(D)$ with $\left.J^{-1}=D\right\}$. Theorem 1 yields the following characterization, for a general $*$-operation, of domains all of whose nonzero integral ideals are $*$-ideals.

Corollary 16. Let $*$ be a star operation on D. Then:

(1) Every $A \in F(D)$ is a star ideal if and only if (a) for any *-ideal $A \in I(D)$ and for any $x \in D \backslash\{0\}$, the sum $A+x D$ is a $*$-ideal and (b) the union of every chain of $*$-ideals in $I(D)$ is again a $*$-ideal.

(2) If $*$ is of finite character, then every $A \in F(D)$ is a *-ideal if and only if the sum $A+x D$ is a $*$-ideal for any $*$-ideal $A \in I(D)$ and for any $x \in D \backslash\{0\}$.

Proof. (1) It is enough to show that every ideal in $I(D)$ is a $*$-ideal because every $A \in F(D)$ can be written as $A=\frac{B}{r}$ where $B \in I(D), r \in D \backslash\{0\}$ and $A=A^{*}$ implies $\frac{B}{r}=\left(\frac{B}{r}\right)^{*}=\frac{B^{*}}{r}$ which gives $B=B^{*}$. Now note that by $\left(*_{1}\right)$ we have that every nonzero principal ideal of $D$ is a $*$-ideal. This takes care of the only requirement of Theorem 1 not mentioned in Corollary 16. To complete the proof, suppose, on the contrary, that there is an ideal $F \in I(D)$ such that $F$ is not a $*$-ideal. Let $S$ be the set of nonzero *-ideals contained in $F ; S$ is partially ordered by inclusion. Obviously, $S$ is nonempty 
and by (b), $S$ is inductive, and so contains a maximal $*$-ideal $M \subseteq F$. Since $F$ is not a *-ideal, there exists $x \in F \backslash M$, and so by (a), $M+x R$ is a $*$-ideal, which contradicts the maximality of $M$.

(2) It is enough to show that if $*$ is of finite character, then part (b) of (1) holds. For this, let $\left\{A_{\alpha}\right\}$ be a chain under inclusion of $*$-ideals and let $A=\cup A_{\alpha}$. To show that $A$ is a $*$-ideal, let $F$ be a finitely generated subideal of $A$. Then $F \subseteq A_{\beta}$ for some $\beta$. But since $A_{\beta}$ is a $*$-ideal, we have $F^{*} \subseteq A_{\beta} \subseteq \cup A_{\alpha}=A$.

Note that Corollary 16 cannot be extended to semistar operations because of the requirement that nonzero principal ideals be $*$-ideals.

We next state a variant of Theorem 1 in the language of star operations for integral domains.

Theorem 17. Let $*$ be a star operation on an integral domain $D$ and let $P$ be a property of *-ideals. Then every nonzero *-ideal of $D$ has property $P$ if and only if the following three conditions hold.

(1) $P$ is true for every principal nonzero ideal in $I(D)$.

(2) If $A \in I(D)$ is a *-ideal with property $P$ and if $x \in D \backslash\{0\}$, then $(A, x)^{*}$ again has property $P$.

(3) The union of any chain of $*$-ideals with property $P$ in $I(D)$ is a *-ideal with property $P$.

The proof is similar to that of Theorem 1, and so we leave it to the reader. Note that if $P$ is any ideal-theoretic property, then an application of Theorem 17 actually serves to characterizes the condition "Every nonzero fractional ideal of $D$ satisfies $P$ ". Indeed, one need only apply Theorem 17 in case $*$ is the trivial star operation, $d$ (given by $A \mapsto A$ ).

The applications of Theorem 17 for nontrivial star operations are somewhat more interesting. However, to see these applications, we need to recall that if $A \in I(D)$ is such that $A=B^{*}$ for some finitely generated $B \in I(D)$, then $A$ is called a $*$-ideal of finite type. Moreover, if there is $C \in F(D)$ such that $(A C)^{*}=D$, we say that $A$ is $*$-invertible. In this case, it is easy to see that $C^{*}=A^{-1}$. If $*$ is of finite type, then $A$ being $*$-invertible requires that both $A^{*}$ and $A^{-1}$ are *-ideals of finite type. Call $D$ a $*$-Prüfer domain if every $A \in f(D)$ is $*$-invertible. A $v$-Prüfer domain is already well known as a $v$-domain, while, for the general case, the $*$-Prüfer domains have been recently studied in [2]. It was established in [2] that a domain $D$ is a $*$-Prüfer domain if and only if the sum of every pair of $*$-invertible ideals of $D$ is a $*$-invertible ideal of $D$. For a star operation $*$ of finite type, a $*$-Prüfer domain is called a $\mathrm{P} * \mathrm{MD}$. Recall, for a finite character star operation $*$, that a domain $D$ is a $\mathrm{P} * \mathrm{MD}$ if every $A \in f(D)$ is $*$-invertible [15]. A PtMD is usually called a PVMD after its original name "Prüfer v-multiplication domain" given in [12]. It had already been established in [20] that $D$ is a PVMD if and only if the sum of every pair of $t$-invertible ideals is $t$-invertible. It can be shown that if $*$ is a star operation of finite type and $A \in F(D)$ is $*$-invertible, then $A$ is $t$-invertible. Therefore, every $\mathrm{P} * \mathrm{MD}$ is a PVMD. 
Corollary 18. Let $*$ be a star operation on a domain D. Then:

(1) $A^{*}$ is of finite type for each $A \in I(D)$ if the union of every chain of integral $*$-ideals of finite type is again a *-ideal of finite type.

(2) Each $A \in I(D)$ is *-invertible if and only if $(a)(A, x)$ is a *-invertible ideal for every *-invertible $A$ in $I(D)$ and every $x \in D \backslash\{0\}$ and $(b)$ every ascending union of *invertible ideals is $*$-invertible. (Indeed, if there is a star operation $*$ such that every $A \in I(D)$ is *-invertible, then $D$ is a particular kind of completely integrally closed integral domain called a*-completely integrally closed integral domain, or *-CICD for short [2]).

(3) The following three conditions are equivalent:

(i) D is *-completely integrally closed;

(ii) The sum of every *-invertible ideal $A$ in $I(D)$ with any nonzero principal ideal is again $a *$-invertible ideal in $I(D)$ and the union of every chain of $*$-invertible ideals in $I(D)$ is *-invertible;

(iii) $D$ is a *-Prüfer domain such that the union of every chain of $*$-invertible ideals in $I(D)$ is again *-invertible.

Proof. (1) will be proved by verifying the three requirements in Theorem 17 . For the first of these, note that every principal nonzero ideal is a $*$-ideal of finite type. To verify the second condition from Theorem 17 , let $A$ be an ideal such that $A^{*}$ is a $*$-ideal of finite type; i.e., there exists a finitely generated ideal $B$ such that $A^{*}=B^{*}$. Then, for any $x \in D \backslash\{0\}$, we have $(A, x)^{*}=\left(A^{*}, x\right)^{*}=\left(B^{*}, x\right)^{*}=(B, x)^{*}$, a $*$-ideal of finite type. The third requirement from Theorem 17 is provided by (1).

We next prove (2). The "only if" assertion is trivial. For the converse, note first that an ideal $A$ being $*$-invertible is equivalent to $A^{*}$ being $*$-invertible (since $D=\left(A A^{-1}\right)^{*}=$ $\left.\left(A^{*} A^{-1}\right)^{*}\right)$. Also, every principal nonzero ideal is a $*$-invertible $*$-ideal. Hence, in view of conditions (a) and (b) of (2), it follows from Theorem 17 (with $P=$ "is *-invertible") that every $*$-ideal ideal of $D$ is $*$-invertible. As noted above, this means that every ideal of $D$ is $*$-invertible.

It remains to prove (3). Note that (i) $\Rightarrow$ (ii) because in a $*$-CICD, every $A \in F(D)$ is $*$-invertible; and (ii) $\Leftrightarrow$ (iii) because $D$ is a $*$-Prüfer domain if and only if every pair of $*$ invertible ideals of $D$ adds to a $*$-invertible ideal. Finally, for (iii) $\Rightarrow$ (i), suppose, on the contrary, that $A$ is an ideal in $I(D)$ such that $A$ is not $*$-invertible. Since $A$ is nonzero, there is at least a nonzero principal ideal in $A$. Let $S$ be the set of $*$-invertible subideals of $A$. We have already noted that $S$ is nonempty. Let $\left\{H_{i}\right\}$ be any chain in $S$. Then by (iii), $\cup H_{i}$ is $*$-invertible. But this means, by Zorn's Lemma, that $S$ contains maximal elements. Let $M$ be one such maximal element of $S$. Because $A$ is not *-invertible, each member of $S$ is a proper subideal of $A$. In particular, $M$ is a proper subideal of $A$. But then there is $x \in A \backslash M$, which makes $M+(x) *$-invertible, because $D$ is a $*$-Prüfer domain. This contradicts the maximality of $M$, to complete the proof. 
Recall that an integral domain $D$ is a Krull domain if (a) $D$ is a locally finite intersection of localizations of $D$ at height one primes and (b) $D_{P}$ is a discrete valuation domain for each height one prime $P$ of $D$. For instance, any UFD is a Krull domain, and so is any integrally closed Noetherian domain. It is well known that an integral domain $D$ is a Krull domain if and only if every nonzero ideal of $D$ is $t$-invertible: see, e.g., [16]. By analogy, we say, for a finite type star operation $*$, that $D$ is a $*$-Krull domain if every nonzero ideal of $D$ is $*$-invertible. Any $*$-Krull domain is obviously a Krull domain.

Corollary 19. Let $*$ be a star operation of finite type on a domain D. Then:

(a) $D$ is a*-Krull domain if and only if $A+B$ is *-invertible for every pair $A, B$ of *-invertible ideals in $I(D)$ with at least one of $A$ and $B$ being principal and the union of every chain of *-invertible ideals is a *-invertible ideal.

(b) $D$ is a Krull domain if and only if $D$ is a PVMD such that the union of every chain of integral $v$-ideals is a t-invertible ideal.

Proof. We shall prove part (a). (Then (b) will follow as the special case of (a) where $*=t$.) The "only if" assertion is obvious. For the converse, note that the given conditions imply that $D$ is a $\mathrm{P} * \mathrm{MD}$. If the assertion fails, there is a nonzero ideal $A$ in $I(D)$ such that $A$ is not a $*$-invertible ideal. Let $S$ be the set of all $*$-invertible ideals contained in $A$. Then $S$ is nonempty, because $A$ has some principal nonzero subideals; also, $S$ contains the finite sums of such, and of course, $S$ can be partially ordered by inclusion. Now, take a chain of members of $S$ and take their union $B$. By hypothesis, $B$ is $*$-invertible. By Zorn's Lemma, $S$ has at least one maximal element, say $J$. Because we have assumed that $A$ is not $*$-invertible, we can pick $x \in A \backslash J$. But then $(J+(x)) \subseteq A$ is $*$-invertible by the hypothesis, thus contradicting the maximality of $J$.

In closing, we reiterate that our general line of argumentation applies to any algebraic setting whose objects are sets with enriched structure. For instance, the analog of Theorem 1 for fields is easily developed and can be seen to have the following application. Let $K$ be a field with algebraic closure $\bar{K}$. Then $K$ is a perfect field if and only if (a) $\mathrm{K}(\mathrm{u})$ is separable over $K$ for each $u \in \bar{K}$ and (b) if $L$ is a separable field extension of $K$ that is contained in $\bar{K}$ and $v \in \bar{K}$, then the field $L(v)$ is also separable over $K$.

\section{References}

[1] T. Akiba, Remarks on flat and relatively seminormal pairs, Kobe J. Math. 6(1989), 217-222.

[2] D. D. Anderson, D. F. Anderson, M. Fontana and M. Zafrullah, On v-domains and star operations, Comm. Algebra, to appear.

[3] A. Ayache and A. Jaballah, Residually algebraic pairs of rings, Math. Z. 225(1997), 49-65.

[4] J. Beachy, Introductory Lectures on Rings and Modules, London Mathematical Society, Student Texts 47, Cambridge University Press, 1999.

[5] H. Cartan and S. Eilenberg, Homological Algebra, Princeton, 1956. 
[6] R. D. Chatham, Going-down pairs of commutative rings, Rend. Circ. Mat. Palermo Serie II 50(2001), 509-542.

[7] D. E. Dobbs, On INC-extensions and polynomials with unit content, Canad. Math. Bull. 23(1980), 37-42.

[8] D. E. Dobbs, Lying-over pairs of commutative rings, Canad. J. Math. 33(1981), 454-475.

[9] D. E. Dobbs and M. Fontana, Going-up, direct limits and universality, Comm. Math. Univ. St. Pauli 33(1984), 191-196.

[10] D. E. Dobbs, M. Fontana and I. J. Papick, Direct limits and going-down, Comm. Math. Univ. St. Pauli 31(1982), 129-135.

[11] D. E. Dobbs and S. B. Mulay, Flat underrings, Arch. Math. 50(1988), 337-341.

[12] R. Gilmer, Multiplicative Ideal Theory, Dekker, New York, 1972.

[13] S. Glaz, Commutative Coherent Rings, Springer-Verlag, Lecture Notes No. 1371, 1989.

[14] W. Heinzer, Integral domains in which each non-zero ideal is divisorial, Mathematika 15(1968), 164-170.

[15] E. Houston, S. Malik and J. Mott, Characterizations of $*$-multiplication domains, Canad. Math. Bull. 27(1)(1984), 28-52.

[16] E. Houston and M. Zafrullah, On t-invertibility II, Comm. Algebra 17(8)(1989), 1955-1969.

[17] F. Kasch, Modules and Rings, (Translated by D. Wallace) London Mathematical Society Monograph No. 17, 1982.

[18] A. Mimouni, Integral domains in which every ideal is a w-ideal, Comm. Algebra 33(5)(2005), 1345-1355.

[19] A. A. Tuganbaev, Semidistributive Modules and Rings,Math. Appl., 449, Kluwer Acad. Publ., Dordrecht, 1998.

[20] M. Zafrullah, Putting t-invertibility to use, Non-Noetherian Commutative Ring Theory, 429-457, Math. Appl., 520, Kluwer Acad. Publ., Dordrecht, 2000.

Department of Mathematics, University of Iowa, Iowa City, Iowa 52242-1419, U.S.A.

E-mail: dan-anderson@uiowa.edu

Department of Mathematics, University of Tennessee, Knoxville, Tennessee 37996-1300, U.S.A.

E-mail: dobbs@math.utk.edu

57 Colgate St., Pocatello, Idaho 83201, U.S.A.

E-mail: zafrullah@lohar.com 